\title{
Three-body confinement force in a realistic constituent quark model
}

\author{
Z. Papp * \\ Institute of Nuclear Research, H-4001 Debrecen, Pf. 51, Hungary \\ Department of Physics and Astronomy, California State University, Long Beach, CA-90840, USA \\ Fl. Stancu ${ }^{\dagger}$ \\ University of Liège, Institute of Physics B5, Sart Tilman, B-4000 Liège 1, Belgium
}

(November 10, 2018)

\begin{abstract}
We show that in realistic constituent quark models based on a two-body confinement interaction color states appear in the middle of the experimentally known spectrum. To avoid this situation we implement a three-body confinement interaction, introduced on an algebraic basis, into a semirelativistic version of the Goldstone boson exchange constituent quark model and by solving the Faddeev equations we show that this interaction can increase the gap between singlet and color states, such as the latter can be ignored and the known baryons can be described as simple $q^{3}$ systems. We analyze the effect of a $\Delta$ - and a Y-shape three-body interaction.
\end{abstract}

PACS: 12.39.-x,12.39.Pn,12.40.Yx

Keywords: Constituent quark models, confinement, three-body interaction

\section{INTRODUCTION}

In this study we are concerned with constituent quark models where the confinement interaction contains the two-body color operator $F_{i} \cdot F_{j}[1]$. This means to assume that the one-gluon exchange picture survives to all distances. There is no real justification for applying this rule to the confinement, except simplicity. This relatively easy model has been widely used in baryon spectroscopy together with a hyperfine interaction generated either by the one-gluon exchange [2] or by the Goldstone boson (pseudoscalar meson) exchange

\footnotetext{
*e-mail address: zpapp@csulb.edu

$\dagger$ e-mail address: fstancu@ulg.ac.be
} 
[3]. The $F_{i} \cdot F_{j}$ model has often been revived as being a convenient framework for studying $q^{2} \bar{q}^{2}$ systems (tetraquarks), see e. g. Ref. [4], $q^{4} \bar{q}$ systems (pentaquarks), see e. g. Ref. [5] or $q^{6}$ system (the nucleon-nucleon problem), see e.g. Ref. [6].

The present work is devoted to baryons, seen as $q^{3}$ systems. The starting point is the observation that the presence of the $F_{i} \cdot F_{j}$ color operator implies that the Hamiltonian, besides color singlets, also possesses color octet and decuplet states at low energies. These states are undesirable. Their existence is in conflict with the experiment and with conclusions drawn from lattice or continuous formulations of QCD in the confining phase. In practice, these states have tacitly been ignored, relying on the assumption that they do not exist. However, as shown below, colored states are found close to singlets in the spectrum of consituent quark models. Therefore they should either be shifted to higher energies, by a change in the dynamics of the quark model, so that they could be ignored, or otherwise transformed into colorless states by coupling to gluons as for example in [7-9], or to color octet sea $q \bar{q}$ pairs as for example in [10].

A solution in the first direction has recently been proposed by Dmitrasinovic [11]. Although $\mathrm{OCD}$ has a local exact $\mathrm{SU}_{C}(3)$ symmetry, in QCD-inspired potential models this symmetry appears as global, by construction. The observation was that by considering only two-body confining forces in a Hamiltonian model implies that the global $\mathrm{SU}_{C}(3)$ symmetry is implemented only in a restricted way, namely the Hamiltonian is expressed in terms of the quadratic (Casimir) invariant operator of SU(3) only. More generally, one could express any quark model Hamiltonian inspired by QCD in terms of every SU(3) invariant operator. Based on this argument, in Ref. [11] a three-quark confining potential that depends on the cubic invariant operator of $\mathrm{SU}(3)$ has been added to the usual two-body confinement. In a qualitative way, it was shown that such an interaction can increase the gap between the singlet and color states, provided its strength has a specific sign and range. Besides its implications on the spectrum of ordinary $q^{3}$ systems, its role in exotic $q^{2} \bar{q}^{2}$ hadrons has also been considered.

The three-body confinement interaction of Ref. [11] has subsequently been analysed in Ref. [12] in the context of a harmonic oscillator confinement where the size of its effect on the gap between the color and singlet states in the spectrum of a $q^{3}$ system has been estimated. Also the $q^{6}$ system with relevance to the nucleon-nucleon problem has been discussed.

Below we first show explicitly how much the singlet and color states of a realistic constituent quark model appear close to each other. Next, by introducing a three-body confinement force related to the cubic invariant of $\mathrm{SU}(3)$, we demonstrate that one can separate them largely enough so that the color states can be ignored. For this purpose, here we consider the semirelativistic constituent quark model of Ref. [13], into which we implement a three-body confinement interaction. By using the Faddeev approach of Ref. [14] we calculate the lowest singlet, octet and decuplet states of a three-quark system, with this additional three-body confinement force.

\section{BASIS STATES}

Here we present the nucleon sector, namely $\mathrm{S}=1 / 2$ and $\mathrm{I}=1 / 2$. The lowest color octet and decuplet $q^{3}$ states compatible with the Pauli principle are displayed in Tables I and II respectively. For orientation, their excitation energy $E_{N}-E_{0}$, estimated in a two-body 
harmonic oscillator confinement, is shown in the last column. In the same harmonic oscillator confinement the first colorless negative parity state is $395 \mathrm{MeV}$ above the ground state. Therefore one can see that the $0^{+}$color octet and decuplet states appear substantially below this negative parity state. In the following we shall consider only the lowest color states, namely the $0^{+}$and $1^{-}$octets and the $0^{+}$decuplet. It is necessary to displace all color states at energies where they can safely be neglected, if the nucleon resonances are to be interpreted as pure $q^{3}$ excitations. This can indeed be achieved by an additional three-body force, as shown below.

\section{THE HAMILTONIAN}

The realistic total Hamiltonian presently under discussion has the form

$$
H=\sum_{i}\left(m_{i}^{2}+p_{i}^{2}\right)^{1 / 2}+V_{2 b}+V_{3 b}+V_{\chi},
$$

where $\vec{p}_{i}$ and $m_{i}$ are the momentum and the constituent mass of the quark $i, V_{2 b}$ the twobody confinement, $V_{3 b}$ the three-body confinement and $V_{\chi}$ the hyperfine interaction. Taking $V_{3 b}=0$ one recovers the chiral constituent quark model of Ref. [13] which is the simplest semirelativistic version (no tensor, no spin-orbit) of the model originally proposed in Ref. [3], where the hyperfine interaction is spin and flavor dependent. The origin of such an interaction is thought to be the pseudoscalar meson exchange between quarks. This model, either in a nonrelativistic or a semirelativistic form, reproduces well the spectra of light baryons and in particular the correct order of positive and negative parity states. It has been shown that the addition of a tensor interaction to the spin-spin interaction in the Goldstone boson exchange model has a minor effect and thus maintains the quality of the spectrum [15]. Therefore, in the present study where the emphasis is made on the confining interaction the tensor force can be ignored. Although we have chosen a particular model, we expect our conclusions to be relevant for any reasonable constituent quark model with three valence quarks. The detailed parametrization of $V_{\chi}$ can be found in Ref. [13] and we do not reproduce it here, the main emphasis being laid on the confinement potential. We use a $V_{2 b}$ term of the form

$$
V_{2 b}=\sum_{i<j} v^{c}\left(r_{i j}\right)\left(\frac{7}{3}+F_{i}^{a} F_{j}^{a}\right)
$$

where $F_{i}^{a}=\frac{1}{2} \lambda_{i}^{a}$ is the color charge operator of the quark $i, \lambda^{a}$ are the Gell-Mann matrices and

$$
v^{c}\left(r_{i j}\right)=V_{0}+\gamma r_{i j},
$$

with $\gamma=2.33 \mathrm{fm}^{-2}$ and $V_{0}=-416 \mathrm{MeV}$ as in Ref. [13]. The color part of $V_{2 b}$ is consistent with Refs. [11,12] but different from that of [13]. It stems from the stability condition of a $q \bar{q}$ pair but it depends on an arbitrary constant which is fixed to $7 / 3$. Anyhow, we rescale this interaction such as to reproduce the spectrum obtained in [13] (see below).

The three-body confinement interaction has the form [11] 


$$
V_{3 b}=V_{i j k}=\mathcal{V}_{i j k} \mathcal{C}_{i j k}
$$

where $\mathcal{V}_{i j k}$ is the radial part and $\mathcal{C}_{i j k}$ is the color operator

$$
\mathcal{C}_{i j k}=d^{a b c} F_{i}^{a} F_{j}^{b} F_{k}^{c}
$$

with $d^{a b c}$ some real constants, symmetric under any permutation of indices and defined by the anticommutator of the Gell-Mann matrices as $\left\{\lambda^{a}, \lambda^{b}\right\}=2 d^{a b c} \lambda^{c}$. The operator (3.5) can be rewritten in terms of the two independent invariant operators of $\mathrm{SU}(3)$ as

$$
\mathcal{C}_{i j k}=\frac{1}{6}\left[C_{i+j+k}^{(3)}-\frac{5}{2} C_{i+j+k}^{(2)}+\frac{20}{3}\right]
$$

where $C^{(2)}$ is the quadratic (Casimir) and $C^{(3)}$ the cubic invariant. The expectation values of (3.6) can thus easily be obtained from the eigenvalue of $C^{(2)}$ and $C^{(3)}$ [16] for any irreducible representation $(\lambda \mu)$ of $\mathrm{SU}(3)$. These eigenvalues are

$$
\left\langle C^{(2)}\right\rangle=\frac{1}{3}\left(\lambda^{2}+\mu^{2}+\lambda \mu+3 \lambda+3 \mu\right)
$$

and

$$
\left\langle C^{(3)}\right\rangle=\frac{1}{18}(\lambda-\mu)(2 \lambda+\mu+3)(\lambda+2 \mu+3) .
$$

Our attempt to choose a form for $\mathcal{V}_{i j k}$ is related to the present knowledge of confinement. Lattice calculations are ambiguous about the static three-quark potentials in baryons. Both Y-shapes [17] and $\Delta$ shapes $[18,19]$ are supported. The difficulty to distinguish between them is due to the fact that the difference between the two gives at most $15 \%$ in the dominant area law for the baryonic potential (for a recent review see e. g. [20]). Note that these results concern color singlet states only while here we deal with both color singlet and octet states. Potentials between static color sources in higher representations of SU(3), including the octet one, do also exist [21]. They satisfy the so-called Casimir scaling which means that potentials between sources in different representations are proportional to each other such as their ratios is given by the respective ratios of the eigenvalues of the corresponding (quadratic) Casimir operators. On the other hand the scaling proportional to the number of fundamental flux tubes embedded into a higher representation coincides with the Casimir scaling. In this situation we deemed reasonable to consider the working Ansatz that $\mathcal{V}_{i j k}$ has either a triangular shape

$$
\mathcal{V}_{i j k}=\gamma c\left[\left|\overrightarrow{r_{i}}-\overrightarrow{r_{j}}\right|+\left|\overrightarrow{r_{j}}-\overrightarrow{r_{k}}\right|+\left|\overrightarrow{r_{k}}-\overrightarrow{r_{i}}\right|\right]
$$

or a Y-shape

$$
\mathcal{V}_{i j k}=\gamma c\left[\left|\overrightarrow{r_{i}}-\overrightarrow{r_{0}}\right|+\left|\overrightarrow{r_{j}}-\overrightarrow{r_{0}}\right|+\left|\overrightarrow{r_{k}}-\overrightarrow{r_{0}}\right|\right] \text {, }
$$

with $\vec{r}_{0}$ the point where the three flux tubes meet such as to satisfy the $\mathrm{SU}_{C}(3)$ gauge invariance (see e. g. [22]). Taking $c=1$ and $\gamma=1 / 2 \sqrt{\sigma}$ with a strength tension $\sqrt{\sigma} \approx 1$ $\mathrm{GeV} \mathrm{fm}^{-1}$ in (3.9), one recovers the linear confinement potential used in realistic consituent quark models as for example the model of Ref. [13] where the form (3.9) is used in conjunction 
with a two-body color operator $F_{i} \cdot F_{j}$ which brings the factor $1 / 2$ in $\gamma$. Taking $c=1$ and $\gamma=\sqrt{\sigma}$ in (3.10) one recovers the confinement potential used for example in Refs. [22], [23] or [24] where no color operator is present in the confinement part, thus one deals with colour singlets only. Here we use the radial form (3.9) or (3.10) in Eq. (3.4), i.e. in conjunction with a three-body color operator. In our calculations the value of $\gamma$ appearing both in (3.9) and (3.10) is taken to be the same as in Eq. (3.3) and the parameter $c$ thus represents the relative strength of the three-body versus the two-body force. By assuming the same triangular shape both in $V_{2 b}$ and $V_{3 b}$ analytic calculations can be carried for a while. In this way in Ref. [11] it was found that $c$ must be located in the interval $-\frac{3}{2}<c<\frac{2}{5}$. The upper limit ensures that the lowest color singlet is below the lowest color octet state. The lower limit was required by the stability condition of the nucleon, $\left\langle V_{2 b}+V_{3 b}\right\rangle>0$. In Ref. [12] some arbitrariness was noticed for the lower bound because this is related to the choice of the color operator in (3.2). However, as the numerical results of Sec. IV will reveal, the above range of $c$ is entirely satisfactory for our discussion of the triangular shape, because the gap of between the colorless and color states will turn out to be large enough. In fact the common conclusion of [11] and [12] was that $c$ must be negative in order to obtain an increase in the gap between the color octet and singlet states in a $q^{3}$ system. Therefore in the calculations below related to the triangular shape, we take

$$
-\frac{3}{2}<c<0 \text {. }
$$

The Hamiltonian (3.1) was solved by using the Faddeev approach of Ref. [14], adequate for confining potentials. The necessary expectation values for the two-body color operator appearing in (3.2) are given in Table III. The expectation values of the three-body color operator (3.6) are taken from [11] or [12]. These are 10/9,-5/36 and 1/9 for the singlet, octet and decuplet $\mathrm{SU}(3)$ states, respectively.

A particular advantage of the Faddeev approach is that the incorporation of permutation symmetry is very easy. For identical quarks, as in this case, the three Faddeev components of the three-quark wave function have the same functional form in their own Jacobi coordinate systems. Therefore the three equations can be reduced to a single one. From the structure of this equation it follows that the correct symmetry of the wave function under the exchange of any two particles is automatically guaranteed if the correct symmetry is implemented in one of the three components. In our calculation a bipolar harmonic basis was used, combined with spin, isospin and color basis states. If we select the basis states such as

$$
(-)^{l+s+i+c}=-1
$$

where $l, s, i$ and $c$ are the relative angular momentum, spin, isospin and color quantum numbers of a two-quark pair, the Pauli principle for the three-quark system is satisfied. We have $(-)^{c}=1$ when $[\tilde{f}]_{C}=[2]$ and $(-)^{c}=-1$ when $[\tilde{f}]_{C}=[11]$, where $[\tilde{f}]_{C}$ is a given partition in the color space. If we denote the total angular momentum by $L$ and the total parity by $P=(-)^{l+\lambda}$, where $\lambda$ is the relative angular momentum of the third particle with respect to the pair, then the lowest color octets must have $L^{P}=0^{+}, 1^{-}$and the lowest decuplet $L^{P}=0^{+}$. In Table IV we show the structure of the lowest color states with the corresponding quantum numbers of their components. This treatment of permutation symmetry as well as the whole numerical procedure were checked against the exact harmonic oscillator results of Ref. [12]. 


\section{RESULTS}

As mentioned above, our purpose is to understand the implications of a three-body color confinement interaction in a realistic model. We look separately at its effect either due to the $\Delta$-shape (3.9), or to the Y-shape (3.10).

\section{A. The $\Delta$-shape.}

This shape is currently used in conjunction with a two-body $F_{i} \cdot F_{j}$ color operator as an approximation to the Y-shape [22]. Here we use it both in $V_{2 b}$ and $V_{3 b}$ as discussed above. Then the contribution of the color part of $V_{2 b}$ and $V_{3 b}$ sum up together to $\chi_{i}(\mathrm{i}=1,8,10)$ as in Eq. (14) of Ref. [12].

$$
\chi_{i}=\left\{\begin{array}{cl}
\frac{5}{3}+\frac{10}{9} c & \mathrm{i}=1 \text { (singlet) } \\
\frac{13}{6}-\frac{5}{36} c & \mathrm{i}=8 \text { (octet) } \\
\frac{8}{3}+\frac{1}{9} c & \mathrm{i}=10 \text { (decuplet) }
\end{array}\right.
$$

In Fig. 1 we show the dependence of some eigenvalues of the Hamiltonian (3.1) as a function of $-c$ for $-1.4 \leq c \leq 0$. The case $c=0$ is the model [13] for which we reproduced the ground state nucleon mass $m_{N}=940 \mathrm{MeV}$, and the resonance masses $\mathrm{N}(1440) 1 / 2^{+}=$ $1459 \mathrm{MeV}, \mathrm{N}(1535) 1 / 2^{-}=1522 \mathrm{MeV}$ and $\mathrm{N}(1710) 1 / 2^{+}=1783 \mathrm{MeV}$. For $c=0$ the lowest color octets $0^{+}$and $1^{-}$acquire the masses $1536 \mathrm{MeV}$ and $1758 \mathrm{MeV}$ respectively and the $0^{+}$ color decuplet has $2077 \mathrm{MeV}$. Thus in a constituent quark model with three valence quarks the color states are so low that they cannot be ignored. If the three-body interaction (3.9) is switched on, the singlet states go substantially down and the color states go slightly up (or down) and a clear gap between the lowest color singlet $\mathrm{S}=1 / 2, \mathrm{I}=1 / 2$ states and the color $q^{3}$ states emerges. This gap increases when $c$ is decreased. At $c \sim-1.4$ it aquires a substantial value of more than $1500 \mathrm{MeV}$.

Note however that the singlet states part of the spectrum shrinks and one has to bring it back close to the experimental spectrum. This can be achieved by a readjustment of the model parametres defined in Ref. [13]. In particular, in order to enlarge the distance between color singlet states one has to increase the strength tension $\gamma$ in (3.3) which will amount to an increase of the gap between the color and colorless states as well. It is then inferred that the gap will be large enough so that color states can safely be neglected.

Before ending this subsection a word should be mentioned about the influence of the choice of the additional constant $7 / 3$ in (3.2) on the results. As shown by Dmitrasinovic [11] the presence of this positive constant is crucial for the stability of a $q \bar{q}$ pair. In Ref. [12] some arbitrariness was noticed regarding this constant. In the $q^{3}$ spectrum, a change in the above additional constant amounts to a shift of the whole spectrum and some change in the slope of the confinement. If one wants to restore the spectrum to its initial form one has 
to change the arbitrary constant $V_{0}$ and the quantity $\gamma$ in Eq. (3.3). For example, if the additional constant is increased, $\gamma$ must be decreased. As a consequence the gap between the color and singlet states decreases. But this can be compensated by further decreasing the lower limit of $c$ below - 1.5, which is allowed in the way discussed in [12].

\section{B. The Y-shape.}

This is a genuine three-body force, both in the coordinate and the color space as well. Then the color part contributions of $V_{2 b}$ and $V_{3 b}$ does not sum up analytically to $\chi_{i}$ as in Eq. (4.1), because the expectation value of the radial part of $V_{2 b}$ is different from that of $V_{3 b}$. Hence the numerical calculation of the entire spectrum is slightly more complicated.

In the Y-shape, the flux tubes meet at $120^{\circ}$ in order to ensure the minimum energy. If one of the interior angles of the flux-tube configuration is greater than $120^{\circ}$ the minimum energy condition cannot be satisfied and the corresponding flux-tube collapses to a point which means that $\vec{r}_{i}=\vec{r}_{0}$ for $i=1,2$ or 3 . Thus the $\mathrm{Y}$ shape moves continuously into a two-legged flux-tube configuration, where the legs meet at an angle greater than $120^{\circ}$. The geometrical arguments are clearly given in Ref. [22] for example.

The spectrum associated to the Y-shape (3.10) is presented in Fig. 2. In this case the lower limit on $c$ imposed by the inequality (3.11) is no more valid but $c$ must remain negative. So we varied the parameter $c$ between zero and -2 . At $c=0$ the spectrum is the same as in Fig. 1.

The trend is similar to the $\Delta$-shape case, but the mass of every colorless state vary more slowly with the strength $|c|$ of the three-body Y-shape force. If one would rescale the $|c|$-axis by a factor $1 / 2$, the $\Delta$ and the Y-shape results would look closer to each other.

To better understand the role of the three-body confinement force we also calculated the root-mean-square radii associated to the states of Fig. 2. These are displayed in Fig. 3. One can see that the quark core radii of the ground state nucleon and its lowest $\mathrm{N}(\mathrm{J}=1 / 2)$ resonances increase slowly with $|c|$. This is obviously the effect of the decrease of the confinement contribution through the addition of the three-body term. On the other hand, the radii of the color states remain practicaly unchanged, similar to their energies.

\section{CONCLUSIONS}

Through the example of the Goldstone boson exchange model [13] we have shown that the spectrum of a realistic constituent quark model with a pairwise color confinement operator and a linearly increasing behaviour can accomodate both singlet and color low-lying $q^{3}$ states. The lowest octet and decuplet color states appear in the middle of the observed spectrum which means they cannot be ignored. In this situation there are two alternatives:

1) To keep simplicity. Then, as shown here, the addition of a three-body confinement interaction can increase the gap between singlet and color states by $1.5 \mathrm{GeV}$ or more. Then the color states can be neglected in calculations and the presently observed baryons can be described as pure $q^{3}$ states. To restore the quality of the spectrum one has to refit 
the parameters of the model, including the strength tension $\gamma$, which has to be increased in order to enlarge the spacing between colorless states. The increase of $\gamma$ will lead to a further increase of the gap between the colorless and color states.

2) If however the color states are maintained low in the spectrum of a Hamiltonian with a pairwise confinement interaction only, one has to give up the simple $q^{3}$ picture of baryons. The color states could give rise to colorless hybrid baryons having singlet-singlet + octet-octet color components either of type $(q q q)(q \bar{q})$ or of type $(q q q) g$. In the context of a potential model based on a one-gluon exchange hyperfine interaction, applied to the study of tetraquarks i. e. $q \bar{q} q \bar{q}$ systems, it has been shown $[4,25,26]$ that there is a strong mixing between states formed of two color singlet and two color octet $(q \bar{q})$ pairs which leads to a substantial lowering of the variational energy. This should also be the case for hybrid baryons where singlet-singlet $(q q q)_{1}(q \bar{q})_{1}$ and octet-octet $(q q q)_{8}(q \bar{q})_{8}$ components could mix substantially. The present study suggests that such hybrids are to be expected in all partial waves if only two-body $F_{i} \cdot F_{j}$ are considered. This is in agreement with the findings of Ref. [10] based on a two-body confinement and a schematic flavor and spin dependent hyperfine interaction model. The subject deserves a separate investigation inasmuch as the hybrid baryons raise a new interest [27].

Acknowledgements. We are most grateful to Stephane Pepin and Jean-Marc Richard for useful suggestions and a careful reading of the manuscript. We are also grateful to Gunnar Bali for useful correspondence on lattice calculations. One of us (Z.P.) acknowledges financial support from the Fond National de la Recherche Scientifique of Belgium and is grateful for warm hospitality at the Theoretical Fundamental Physics Laboratory of the University of Liège. This work has been partially supported by OTKA Grants T026233 and T029003. 


\section{REFERENCES}

[1] O. W. Greenberg and H. J. Lipkin, Nucl. Phys. A370 (1981) 349.

[2] A. de Rujula, H. Georgi and S. L. Glashow, Phys. Rev. D12 (1975) 147.

[3] L.Ya. Glozman and D.O. Riska, Phys. Rep. 268 (1996) 263.

[4] J. Weinstein and N. Isgur, Phys. Rev. D27 (1983) 588; ibid D41 (1990) 2236.

[5] Fl. Stancu, Phys. Rev. D58 (1998) 111501.

[6] M. Harvey, Nucl. Phys. A352 (1981) 301; ibid. A352 (1981) 326.

[7] H. J. Lipkin, Phys. Lett B251 (1990) 613.

[8] T. P. Cheng and Ling-Fong Li, Phys. Rev. Lett. 74 (1995) 2872.

[9] J. Linde, T. Ohlsson and H. Snellman, Phys. Rev. D57 (1998) 452.

[10] C. Helminen and D. O. Riska, Nucl. Phys. A699 (2002) 624.

[11] V. Dmitrasinovic, Phys. Lett B499 (2001) 135.

[12] S. Pepin and Fl. Stancu, Phys. Rev. D65 (2002) 054032.

[13] L. Ya. Glozman, Z. Papp, W. Plessas, K. Varga and R. F. Wagenbrunn, Phys. Rev. C57 (1998) 3406; L. Ya. Glozman, W. Plessas, K. Varga and R. F. Wagenbrunn, Nucl. Phys. A631 (1998) 469c; Phys. Rev. D58 (1998) 094030.

[14] Z. Papp, A. Krassnigg and W. Plessas, Phys. Rev. C62 (2000) 044004.

[15] W. Plessas et al. Proceedings of the Second International Conference on Perspectives in Hadronic Physics, eds. S. Boffi et al. World Scientific Singapore 2000) p. 136

[16] Fl. Stancu, Group Theory in Subnuclear Physics, (Oxford University Press, Oxford, 1996).

[17] T. T. Takahashi, H. Matsufuru, Y. Nemoto and H. Suganuma, Phys. Rev. Lett. 86 (2001) 18.

[18] G. S. Bali, Phys. Rep. 343 (2001) 1.

[19] C. Alexandrou, Ph. de Forcrand and A. Tsapalis, Phys. Rev. D65 (2002) 054503.

[20] G. S. Bali, Proceedings of the NSTAR Workshop on the Physics of Excited Nucleons, Pittsburgh, Pennsylvania, 9-12 October 2002 (arXiv:nucl-th/0302039).

[21] G. S. Bali, Phys. Rev. D62 (2000) 114503.

[22] J. Carlson, J. Kogut and V. R. Pandharipande, Phys. Rev. D27 (1983) 233.

[23] R. Sartor and Fl. Stancu, Phys. Rev. D31 (1985) 128; Phys. Rev. D34 (1986) 3405.

[24] S. Capstick and N. Isgur, Phys. Rev. D34 (1986) 2809.

[25] S. Zouzou, B. Silvestre-Brac, C. Gignoux and J. -M. Richard, Z. Phys C30 (1986) 457.

[26] D. M. Brink and Fl. Stancu, Phys. Rev. D49 (1994) 4665.

[27] P. R. Page, arXiv:nucl-th/0204031. 


\section{TABLES}

TABLE I. Three-quark color octet states $[21]_{C}$ compatible with the Pauli principle. The first column gives the orbital angular momentum $L$ and parity $P$, the second, third and fourth columns give the permutation symmetry in the orbital, spin-isospin ad spin-isospin-color spaces respectively and the last column gives an estimates of the excitation energy $E_{N}-E_{0}=(N+3) \hbar \omega\left[\bar{\chi}_{8}\right]^{1 / 2}-3 \hbar \omega$ of each state for a harmonic oscillator two-body confinement with $N$ quanta, $\omega / c=2 \mathrm{fm}^{-1}$ and $\bar{\chi}_{8}=\chi_{8} / \chi_{1}$ with $\chi_{8}$ and $\chi_{1}$ from Eq. (4.1) at $c=0$.

\begin{tabular}{lccccc}
\hline \hline \hline$L^{P}$ & {$[f]_{O}$} & {$[f]_{I S}$} & {$[f]_{I S C}$} & $\mathrm{~N}$ & $\begin{array}{c}E_{N}-E_{0} \\
(\mathrm{MeV})\end{array}$ \\
\hline $0^{+}$ & {$[3]$} & {$[21]$} & {$\left[1^{3}\right]$} & 0 & 166 \\
$1^{-}$ & {$[21]$} & {$[3]$} & {$[21]$} & 1 & 616 \\
$1^{-}$ & {$[21]$} & {$[21]$} & {$[21]$} & 1 & 616 \\
$1^{-}$ & {$[21]$} & {$\left[1^{3}\right]$} & {$[21]$} & 1 & 616 \\
$1^{+}$ & {$\left[1^{3}\right]$} & {$[21]$} & {$[3]$} & 2 & 1066 \\
\hline \hline
\end{tabular}

TABLE II. Same as Table I but for color decuplet $[3]_{C}$ states where $E_{N}-E_{0}=(N+3) \hbar \omega\left[\bar{\chi}_{10}\right]^{1 / 2}-3 \hbar \omega$ with $\bar{\chi}_{10}=\chi_{10} / \chi_{1}$ where $\chi_{10}$ and $\chi_{1}$ are from Eq. (4.1) at $c=0$.

\begin{tabular}{lccccc}
\hline \hline$L^{P}$ & {$[f]_{O}$} & {$[f]_{I S}$} & {$[f]_{I S C}$} & $\mathrm{~N}$ & $\begin{array}{c}E_{N}-E_{0} \\
(\mathrm{MeV})\end{array}$ \\
\hline $0^{+}$ & {$[3]$} & {$\left[1^{3}\right]$} & {$\left[1^{3}\right]$} & 0 & 314 \\
$1^{-}$ & {$[21]$} & {$[21]$} & {$[21]$} & 1 & 814 \\
$1^{+}$ & {$\left[1^{3}\right]$} & {$[3]$} & {$[3]$} & 2 & 1312 \\
\hline \hline
\end{tabular}

TABLE III. The expectation values of the two-body color operator $O_{i j}=\frac{7}{3}+F_{i} \cdot F_{j}$ of Eq. (3.2) $(i<j)$ between three-quark states $\left|\left(c_{2} c_{3}\right)[\tilde{f}]_{C} ; c_{1}[f]_{C}\right\rangle$ required in the Faddeev calculations. The particles 2 and 3 are first coupled to a symmetric [2] or an antisymmetric $\left[1^{2}\right]$ state and next to particle 1 to a total color symmetry $[f]_{C}$.

\begin{tabular}{lccc}
\hline \hline$\overline{\tilde{f}]_{C}}$ & {$[f]_{C}$} & Color operator & Expectation value \\
\hline$[2]$ & {$[3]$} & $O_{i j}$ & $8 / 3$ \\
{$[2]$} & {$[21]$} & $O_{23}$ & $8 / 3$ \\
{$[2]$} & {$[21]$} & $O_{12}$ & $23 / 12$ \\
{$[2]$} & {$[21]$} & $O_{13}$ & $23 / 12$ \\
{$[11]$} & {$[21]$} & $O_{23}$ & $5 / 3$ \\
{$[11]$} & {$[21]$} & $O_{12}$ & $29 / 12$ \\
{$[11]$} & {$[21]$} & $O_{13}$ & $29 / 12$ \\
{$[11]$} & {$\left[1^{3}\right]$} & $O_{i j}$ & $5 / 3$ \\
\hline \hline
\end{tabular}


TABLE IV. The quantum numbers of the components of a $q^{3}$ totally antisymmetric state of color symmetry $[f]_{C}$, total angular momentum $L$, total spin $\mathrm{S}=1 / 2$, and total isospin $\mathrm{I}=1 / 2$, compatible with the Pauli principle, as used in the Faddeev calculations. Here $l, s, i$ and $[\tilde{f}]_{C}$ are the relative angular momentum, spin, isospin and color quantum numbers of a two-quark pair and $\lambda$ is the relative angular momentum of the third particle with respect to the pair.

\begin{tabular}{lllllll}
\hline \hline$\overline{[f]_{C}}$ & $L$ & $l$ & $\lambda$ & $s$ & $i$ & {$[\tilde{f}]_{C}$} \\
\hline$[21]$ & 0 & 0 & 0 & 0 & 0 & {$[11]$} \\
& 0 & 0 & 1 & 1 & {$[11]$} \\
& 1 & 1 & 1 & 0 & {$[11]$} \\
& 1 & 1 & 0 & 1 & {$[11]$} \\
& 0 & 0 & 1 & 0 & {$[2]$} \\
& 0 & 0 & 0 & 1 & {$[2]$} \\
& 1 & 1 & 0 & 0 & {$[2]$} \\
\hline$[21]$ & 1 & 1 & 1 & 1 & {$[11]$} \\
& & 0 & 1 & 0 & 0 & {$[11]$} \\
& 0 & 1 & 1 & 1 & {$[11]$} \\
& 1 & 0 & 0 & 1 & {$[2]$} \\
& & 1 & 0 & 1 & 0 & {$[2]$} \\
& 1 & 0 & 0 & 0 & {$[2]$} \\
& 1 & 0 & 1 & 1 & {$[2]$} \\
\hline$[3]$ & 1 & 1 & 0 & 1 & {$[2]$} \\
& & 0 & 1 & 1 & 0 & {$[2]$} \\
\hline \hline
\end{tabular}




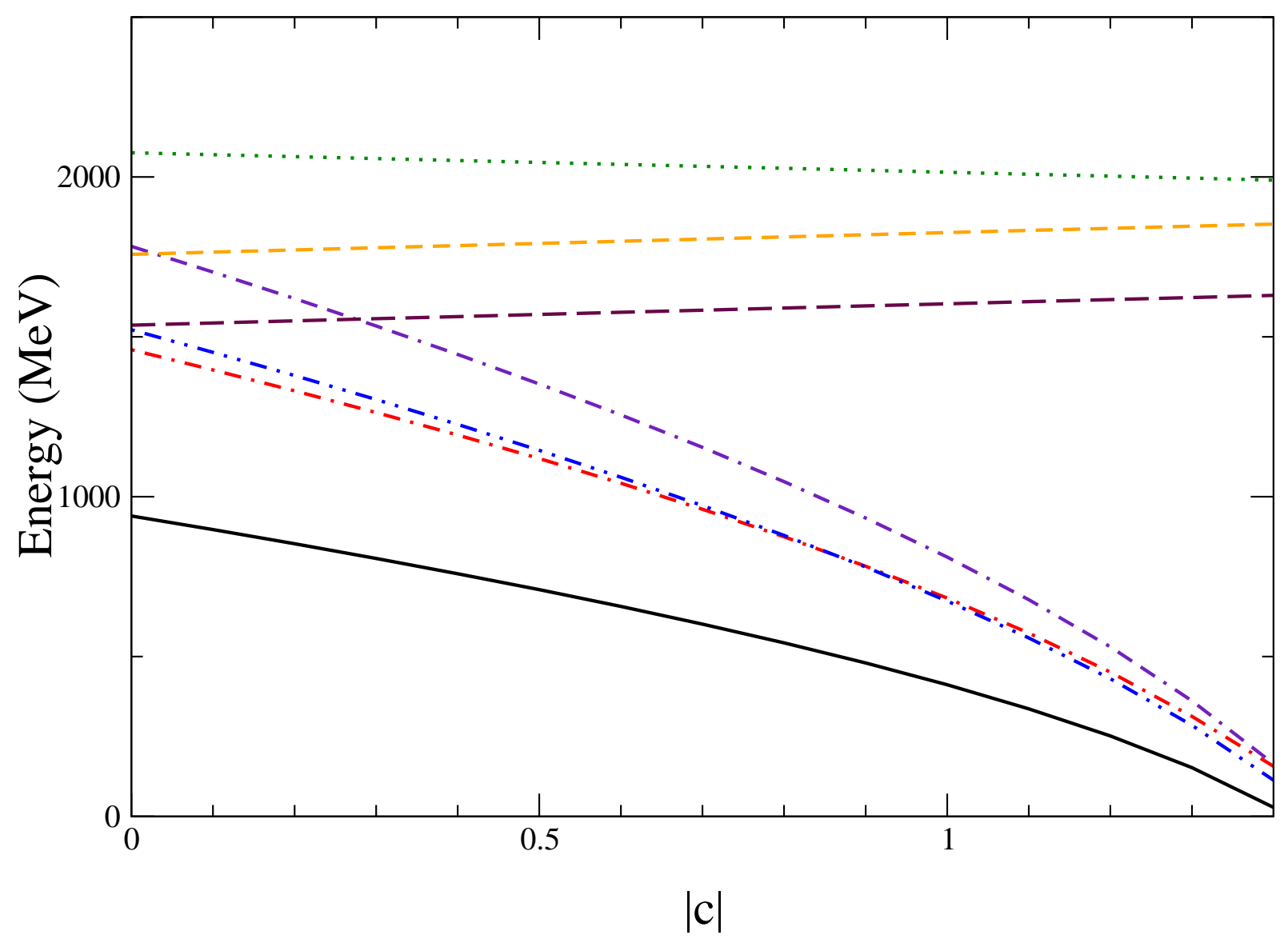

FIG. 1 . $\mathrm{S}=1 / 2, \mathrm{I}=1 / 2$ eigenvalues of the Hamiltonian (3.1) with the $\Delta$-shape three-body interaction (3.9) as a function of $-c$. The solid line is the ground state nucleon, the dash-dotted line the $\mathrm{N}$ (1440) resonance (Roper), the double-dot-dashed line the lowest negative parity state $\mathrm{N}(1535)$, the double-dash-dotted line the $\mathrm{N}(1710)$ resonance. The color octets $0^{+}$and $1^{-}$are represented by long- and short-dashed lines respectively and the $0^{+}$color decuplet by a dotted line. 


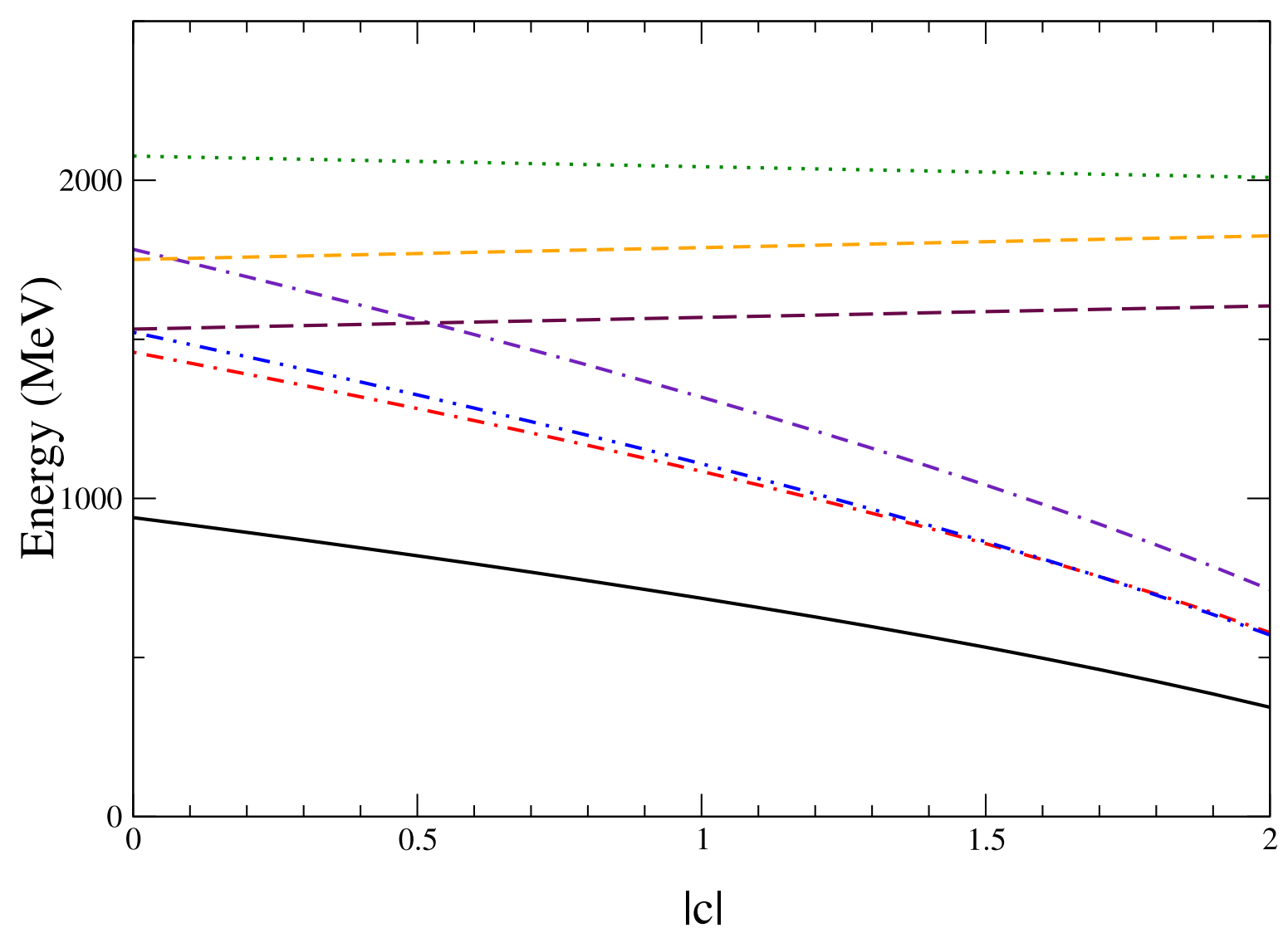

FIG. 2. Same as Fig. 1 but for the Y-shape interaction (3.10). 


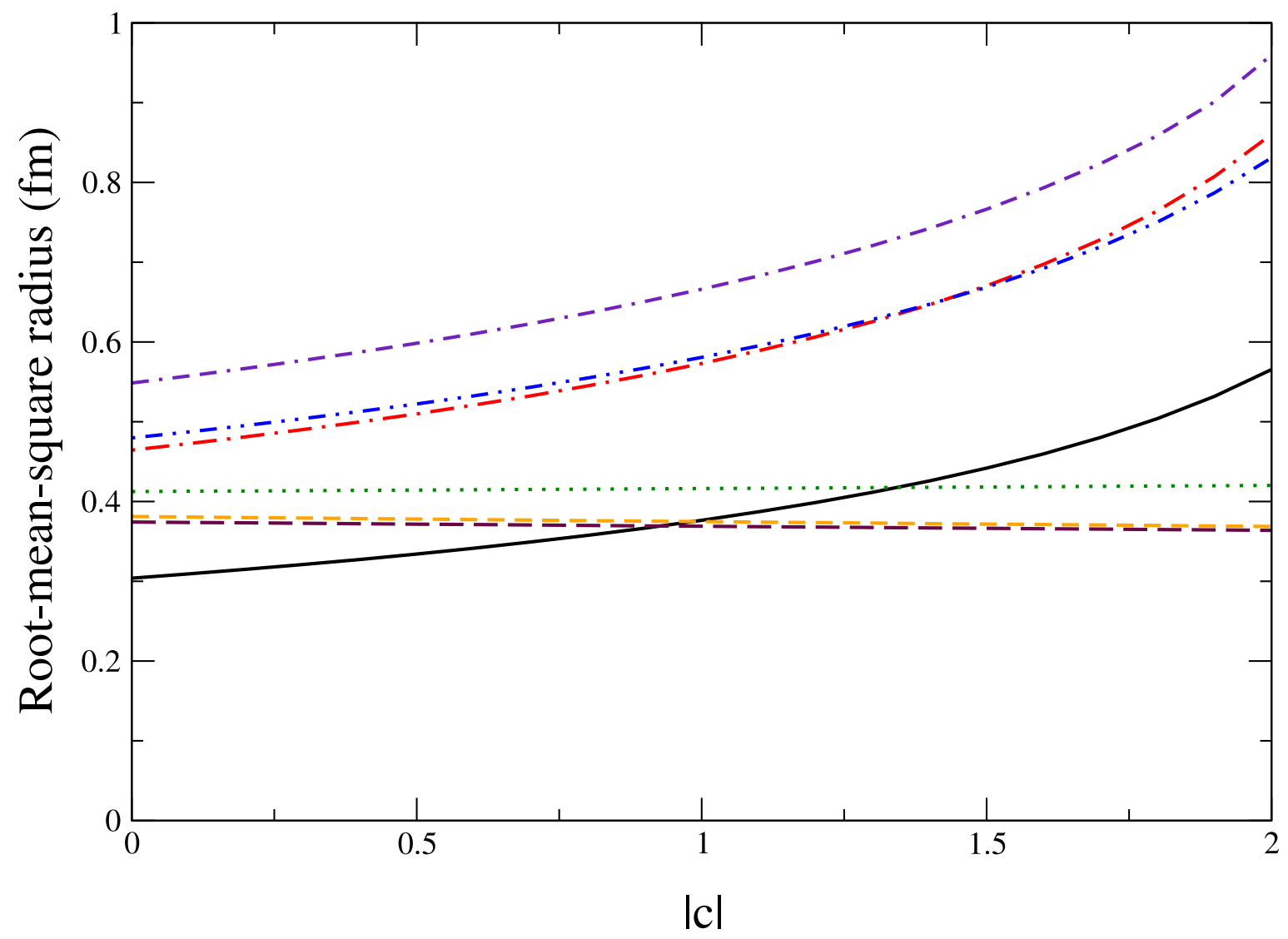

FIG. 3. Root mean square radii of states of Fig. 2. 